\title{
Optical communications beyond orbital angular momentum
}

Rosales-Guzmán, Carmelo, Trichili, Abderrahmen, Dudley, Angela, Ndagano, Bienvenu, Ben Salem, Amine, et al.

Carmelo Rosales-Guzmán, Abderrahmen Trichili, Angela Dudley, Bienvenu Ndagano, Amine Ben Salem, Mourad Zghal, Andrew Forbes, "Optical communications beyond orbital angular momentum," Proc. SPIE 9950, Laser Beam Shaping XVII, 99500C (27 September 2016); doi: 10.1117/12.2238416

EDEent: SPIE Optical Engineering + Applications, 2016, San Diego, California, United States 


\title{
Holographic toolkit for optical communication beyond orbital angular momentum
}

\author{
Carmelo Rosales-Guzmán ${ }^{\mathrm{a}}$, Abderrahmen Trichili ${ }^{\mathrm{b}}$, Angela Dudley ${ }^{\mathrm{a}, \mathrm{c}}$, Bienvenu Ndagano ${ }^{\mathrm{a}}$, \\ Amine Ben Salem ${ }^{\mathrm{b}}$, Mourad Zghal ${ }^{\mathrm{b}, \mathrm{c}}$, and Andrew Forbes ${ }^{\mathrm{a}}$

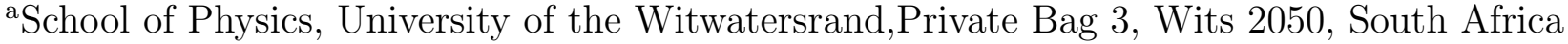 \\ ${ }^{b}$ University of Carthage, Engineering School of Communication of Tunis (Sup'Com), \\ GreS'Com Laboratory, Ghazala Technopark, 2083, Ariana,Tunisia \\ ${ }^{\mathrm{c}}$ CSIR, PO Box 395, Pretoria 0001, South Africa \\ dnstitut Mines-Télécom/Télécom SudParis, 9 rue Charles Fourier, 91011 Evry, France
}

\begin{abstract}
Current optical communication technologies are predicted to face a bandwidth capacity limit in the near future. The nature of the limitation is fundamental rather than technological and is set by nonlinearities in optical fibers. One solution, suggested over 30 years ago, comprises the use of spatial modes of light as information carriers. Along this direction, light beams endowed with orbital angular momentum (OAM) have been demonstrated as potential information carriers in both, free space and fibres. However, recent studies suggest that purely OAM modes does not increase the bandwidth of optical communication systems. In fact, in all work to date, only the azimuthal component of transverse spatial modes has been used. Crucially, all transverse spatial modes require two degrees of freedom to be described; in the context of Laguerre-Gaussian $\left(L G_{p \ell}\right)$ beams these are azimuthal $(\ell)$ and radial $(p)$, the former responsible for OAM. Here, we demonstrate a technique where both degrees of freedom of LG modes are used as information carrier over free space. We transfer images encoded using 100 spatial modes in three wavelengths as our basis, and employ a spatial demultiplexing scheme that detects all 100 modes simultaneously. Our scheme is a hybrid of MIMO and SMM, and serves as a proof-of-principle demonstration. The cross-talk between the modes is small and independent of whether OAM modes are used or not.
\end{abstract}

Keywords: Laguerre-Gaussian modes, Optical communications, orbital angular momentum

\section{INTRODUCTION}

The dramatic growth of data traffic has changed the landscape of modern telecommunication networks. Exciting progress in polarization division multiplexing (PDM) and wavelength division multiplexing (WDM) technologies as well as the use of high order modulation formats have contributed significantly to the increase in optical communication bit rates. ${ }^{1-3}$ However, it has been predicted that current technologies will reach a capacity limit in the near future, a fundamental limit imposed by nonlinearities in optical fibres. ${ }^{4}$ One potential solution to eventually cope with this problem is Space-Division Multiplexing (SDM). ${ }^{5-7}$ Of particular interest in SDM is Mode-Division Multiplexing (MDM), a technique based in the use of orthogonal modes, first suggested in 1982 to increase the bandwidth capacity in optical fibre. ${ }^{8}$ In MDM each mode can carry an independent data stream, thereby increasing the capacity and spectral efficiency by a factor equal to the number of modes used. ${ }^{9-12}$ Along this line, Orbital Angular Momentum (OAM) has received special attention since its first demonstration in 1994. ${ }^{13}$ OAM multiplexing techniques have been extensively explored in both optical fibres and free space. ${ }^{14-20}$ Nevertheless, the true capacity limits on OAM-Division multiplexing are still controversial. ${ }^{21,22}$

The practical implementation of MDM systems requires enabling new mode multiplexing and demultiplexing technologies. Hence, huge efforts have been made along this direction to provide with these technologies, specially through the use of Spatial Light Modulators (SLMs). ${ }^{23-25}$ In this study, we focus on further increasing the

Further author information: (Send correspondence to Carmelo Rosales-Guzmán)

CRG: E-mail: carmelo.rosalesguzman@wits.ac.za

Laser Beam Shaping XVII, edited by Andrew Forbes, Todd E. Lizotte, Proc. of SPIE Vol. 9950, 99500C

(C) 2016 SPIE $\cdot$ CCC code: $0277-786 X / 16 / \$ 18 \cdot$ doi: $10.1117 / 12.2238416$

Proc. of SPIE Vol. $995099500 \mathrm{C}-1$ 
number of multiplexed spatial modes to achieve higher transmission optical free space capacity using computer generated holograms. Here we demonstrate a novel experimental technique for information encoding using Laguerre-Gaussian (LG) modes.

The azimuthal dependence of LG modes has been extensively used in a variety of applications in fields as diverse as, quantum optics, optical activity, laser remote sensing, fluid dynamics and optical manipulations to mention just a few. ${ }^{26-32}$ Crucially, LG modes have two degrees of freedom: an azimuthal, related to the OAM content, and a radial. However, in optical communication only the azimuthal component has been used, leaving the radial component unexplored. Here, we describe a novel approach, that comprises the use of both degrees of freedom, for free space optical communications. ${ }^{33}$ In a proof-of-concept experiment we successfully transmitted grayscale and coloured RGB images using over 100 modes. For this, 35 spatial LG modes where generated and multiplexed in three different wavelengths. Crucially, all modes can be generated and detected in real time using a single SLM and a CCD camera. These demonstrations sets the basis to further increasing the capacity in both free-space and optical communications systems. We further believe that the detection concept can be implemented using other spatial mode basis, as for example Hermite-Gaussian.

\section{RESULTS.}

Laguerre-Gaussian modes of radial order $p$ and azimuthal order $\ell$ at its waist plane $(z=0)$ are described by:

$$
\mathrm{LG}_{p \ell}(r, \phi)=\sqrt{\frac{2 p !}{\pi w_{0}^{2}(p+|\ell|) !}} \times\left(\frac{\sqrt{2} r}{w_{0}}\right)^{|\ell|} L_{p}^{|\ell|}\left(\frac{2 r^{2}}{w_{0}^{2}}\right) \exp \left(-\frac{r^{2}}{w_{0}^{2}}\right) \exp (i \ell \phi)
$$

where $(r, \phi)$ are the transverse coordinates, $L_{p}^{|\ell|}$ is the generalised Laguerre polynomial and $w_{0}$ is a scalar parameter corresponding to the Gaussian (fundamental mode) radius. The mode size is a function of the indices and is given by $w_{p \ell}=w_{0} \sqrt{2 p+|\ell|+1}$. Such modes are shape invariant during propagation and reduce to the special case of the Gaussian beam when $p=\ell=0$. We densely pack the available mode space in our multiplexing and demultiplexing scheme, shown in Fig. 1, but making use of indices in both the radial and azimuthal degrees of freedom, and without any gap in indices, from $p=[0,4]$ and $\ell=[-3,4]$. In the first step we multiplex these modes using a spatial light modulator. The encoding is done so that the holograms are wavelength independent (see Methods) so that we can add wavelength as a third degree of freedom. The multiplexed beam was then propagated to a second SLM where it was demultiplexed into 105 channels and detected on a single CCD camera. The creation and detection can be done with very high fidelity so long as care is taken with the hologram generation (see Methods). Figure 2 shows the crosstalk between the input and output modes, showing very good mode detection fidelity.

In this study we consider several encoding methods, as illustrated in Fig. 3. In the first we employ single bit encoding, where each mode is assigned a particular grayscale. In the second we implement RGB encoding, where the three primary colours are used to transfer information through modes of light. In the third we implement multi-bit encoding. In this scheme, 256 levels of contrast can be achieved with just eight modes $\left(2^{8}=256\right)$, which together with assigning modes to represent the primary colours allows high contrast colour images to be sent. The results for each are shown alongside the encoding method. In all cases the fidelity of transfer was very high, exceeding $98 \%$.

\section{DISCUSSION.}

While we have demonstrated the encoding with Laguerre-Gaussian modes, it is also possible to use another mode set: Hermite-Gaussian modes in rectangular symmetry or Bessel-Gaussian modes, for which tools already exist for separating the radial and azimuthal components. ${ }^{34,35}$

Note that while we have used example encoding methods to illustrate the robustness of our link, in a standard optical communication system the modes would be channels, modulated with conventional technology. In other words, the two degrees of freedom of the LG modes with an additional degree of freedom from the wavelength multiply the overall transmission capacity of the communication system by $3 \times 35$ orders of magnitude. With 


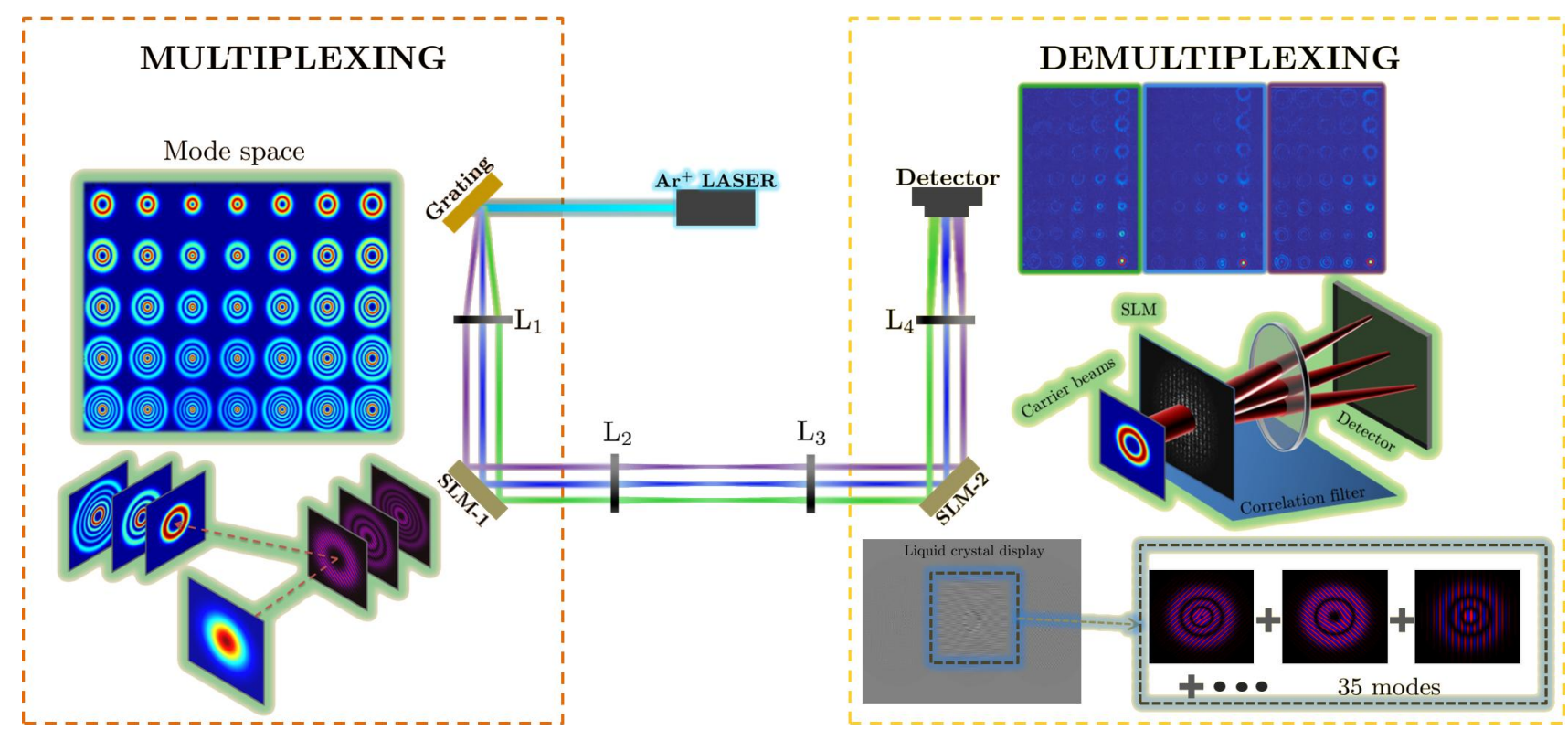

Figure 1. Schematic of the experimental setup. Our experimental setup consist of two stages: MULTIPLEXING and DEMULTIPLEXING. For the first stage we used a continuum linearly-polarized Argon Ion laser. The different components of the continuum laser were separated using a grating, three of this components with wavelengths $\lambda_{1}=457$ $\mathrm{nm}, \lambda_{2}=488 \mathrm{~nm}$ and $\lambda_{3}=514 \mathrm{~nm}$ were redirected to a HoloEye Pluto Spatial Light Modulator (SLM-1). The SLM was split into three independent screens, one for each wavelength, and addressed with holograms to produce the set of thirty five $\mathrm{LG}_{p \ell}$ modes shown in Mode space inset. The information was encoded into the hundred and five options given by the combinations of the three wavelengths and the thirty five different modes. The carrier modes were propagated through free space and detected in the second stage using modal decomposition. This was done by passing the carrier beams trough a modal filter. The modal filter consists of a hologram encoded onto SLM-2 where the complex conjugated of all thirty five modes was multiplexed with different carrier frequencies. The far field of this projection, achieved with a lens, was captured on a CCD camera (see top right inset). An on-axis intensity signifies the presence of a particular mode while a null its absence.

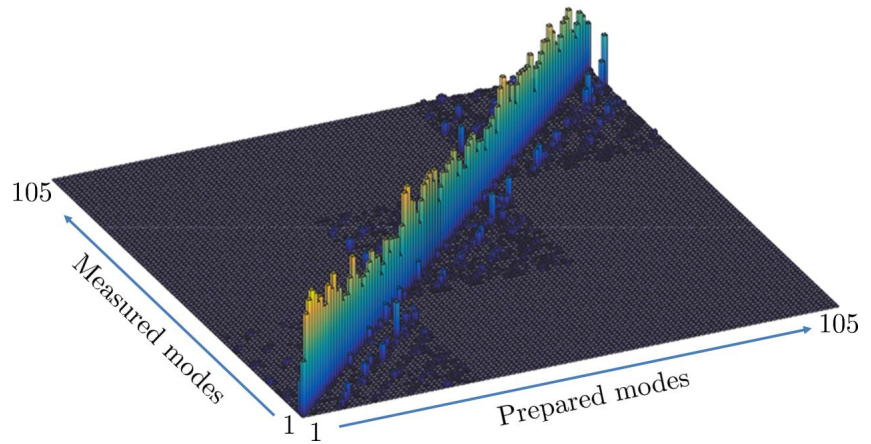

Figure 2. Cross Talk. For each input mode (prepared mode) we measure the on-axis intensity distribution for all hundred and five modes (measured modes). In all cases the measured mode with the highest intensity coincides with the input mode. Hence our detection system is capable to detect the input mode with high accuracy.

the ability of employing 105 spatial modes able to convey independent information signals the CCD detector in this case can be replaced by 105 photodetectors to recover the initially encoded information. One of the key motivation of considering such a decoding technique is its simplicity linked to the dynamic generation of customized digital hologram. Furthermore, the designed correlation filters are wavelength insensitive which allow the technique to operate in large spectrum. However, existing mode (de) multiplexer are extremly wavelength sensitive such as photonic lantern. We believe that this approach could be potentailly extended to several wavelengths and the number of the modes could increase if we employ higher resolution SLMs. 

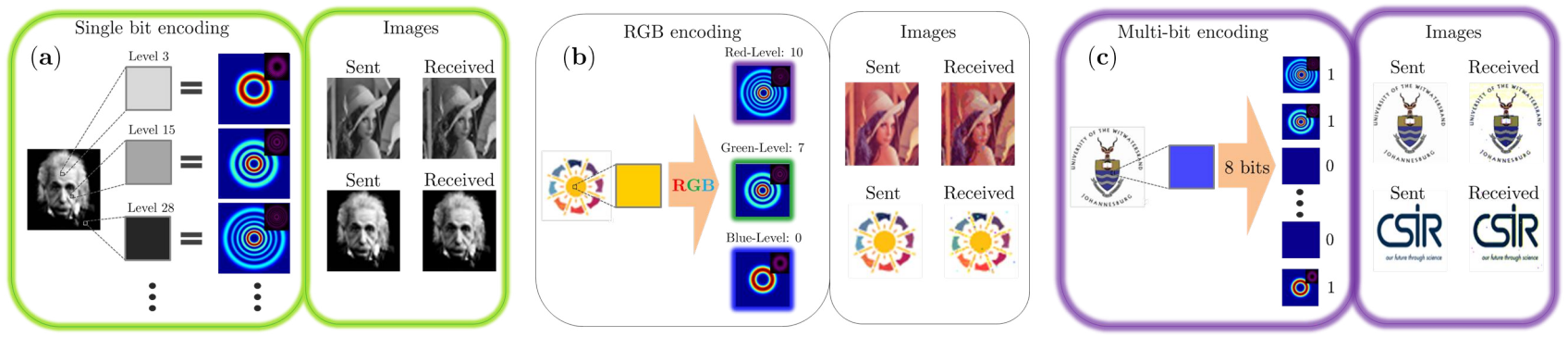

Figure 3. Information encoding. (a) Gray images were encoded in two different ways, in a first approach we send pixel by pixel. For this we assigned a specific $\mathrm{LG}_{p \ell}$ mode and a specific wavelength to the gray level of each pixel, reaching in total hundred five different levels of gray. In a second approach we sent three pixels at the same time by assigning each pixel to each wavelength. With this approach we can increase the speed of information transfer at the expense of decreasing the levels of gray. (b) RGB images can be encoded in a similar way by decomposing each pixel into its three RGB components in such a way that $\lambda_{1}$ carries the information of the different levels of red, $\lambda_{2}$ the different levels of green and $\lambda_{3}$ the different levels of blue. (c) We also encoded information using an 8-bit approach. For this, the gray level of each pixel was first converted to an 8-digit binary number. An specific $\mathrm{LG}_{p \ell}$ mode was assigned to each digit. The hologram encoded in SLM-1 consisted of a superposition of all the modes whose digit value is one. To apply this technique for an RGB image, each colour intensity is encoded with the same manner as a gray-scale image but in a particular wavelength.

In summary, we have presented a novel technique to encode and decode information using high order LG modes. We showed a real time mode identification method from a set of more than 100 modes in three distincts wavelengths by making use of a single digital hologram. The presented results will surely be of relevance to those working on mode division multiplexing communication in free space as well as in optical fibers.

\section{METHODS}

Experimental details. The source, a continuum linearly-polarized Argon Ion laser (Laser Physics: 457-514 $\mathrm{nm})$, was expanded and collimated by a telescope $\left(f_{1}=50 \mathrm{~mm}\right.$ and $\left.f_{2}=300 \mathrm{~mm}\right)$ to approximate a plane wave. Afterwards it was decomposed into its different wavelengths by means of a grating. Three of these components $\left(\lambda_{1}=457 \mathrm{~nm}, \lambda_{2}=488 \mathrm{~nm}\right.$ and $\left.\lambda_{3}=514 \mathrm{~nm}\right)$, propagating almost parallel to each other, where redirected to a HoloEye Pluto Spatial Light Modulator (SLM, $1080 \times 1920$ pixels) with a resolution of $8 \mu \mathrm{m}$ per pixel. The SLM was split into three thirds, one for each beam, each of which could be manipulated independently. Each third was addressed with holograms representing the set of Laguerre-Gaussian modes $\left(\mathrm{LG}_{p \ell}\right)$, where $p$ is the radial index and $\ell$ the azimuthal index. For this experiment we used 35 different modes, resulting from the combinations of $p=[0,1,2,3,4]$ and $\ell=[-3,-2,-1,1,2,3,4]$. It should be stressed that the selection of the modes was made arbitrary and does not exclude any other combinations of LG modes. These modes were encoded via complex amplitude modulation and only the first diffracted order from each third of the SLM was used. The information encoding was achieved by specifically assigning a particular mode and wave length to a gray level, for example the mode $\mathrm{LG}_{0-3}$ produced by $\lambda_{1}$ can be assigned to the lowest gray level and the mode $\mathrm{LG}_{44}$ generated with $\lambda_{3}$ to the highest. For the case of RGB images, the different Red labels can be associated to the 35 different modes and to a particular wavelength, let's say $\lambda_{1}$ and similarly the Blue and Green levels can be associated to $\lambda_{2}$ and $\lambda_{3}$.

The information decoding was achieved using modal decomposition, for this, the three beams were imaged onto a second SLM using one to one image system $\left(f_{3}=150 \mathrm{~mm}\right)$. The second SLM was split into three thirds, each third was addressed with a superposition of the 35 different modes, each with different spatial carrier frequencies. The Fourier transform of the field at the plane of the SLM (i.e. the superposition of the thirty five modes) was achieved with the use of a lens $f=20 \mathrm{~mm}$ and the first diffraction order captured on a CCD camera (Point Grey Flea3 Mono USB3 $1280 \times 960$ ). In our experiment, it was necessary to compensate for small spherical aberrations, this was done by digitally encoding a cylindrical lens on the second SLM.

Hologram generation and Spatial-Multiplexing. In order to encode the LG modes [described mathematically in equation (1)] we make use of the complex-amplitude approach proposed by Arrizón et al in. ${ }^{36}$ We program 
a complex valued function, $T(\mathbf{r})=A(\mathbf{r}) \exp [i \Phi(\mathbf{r})]$ where $A \in[0,1]$ and $\Phi \in[-\pi, \pi]$ into a phase-only digital hologram $H(\mathbf{r})=\exp [i \phi(\mathbf{r})]$ where $\phi(A, \Phi)$ is the encoded phase modulation. This phase modulation is determined from the following ansatz $\phi(A, \Phi)=f(A) \sin (\Phi)$ where $f(A)$ is obtained from inverting $J_{1}[f(A)]=0.58 A$ where $J_{1}(x)$ is the first-order Bessel function. To separate the desired mode from the undiffracted mode (i.e. the zero order), a sinusoidal grating was superimposed with the encoded phase modulation $\phi(A, \Phi)$. Examples of encoded phase profiles $\phi(A, \Phi)$ are presented in the first column of fig. 4 (a) together with their experimentally recorded (middle column) and theoretically calculated (last column) intensity images obtained in the far-field plane of the first SLM.

The encoded phase profiles can be modified by implementing angular multiplexing to allow more than one LG mode to be created and measured via modal decomposition. This involves superimposing a series of single transmission functions $T_{n}(\mathbf{r})$ each multiplied with a unique carrier frequency $\mathbf{K}_{n}$ [e.g. the three holograms given in the top row of fig. 4 (b)] to produce a final transmission function $T(\mathbf{r})$ [bottom, left image of fig. 4 (b)],

$$
T(\mathbf{r})=\sum_{n=1}^{N} T_{n}(\mathbf{r}) \exp \left(i \mathbf{K}_{n} \mathbf{r}\right)
$$

where $N$ is the maximum number of multiplexed modes. In the Fourier plane the carrier frequencies $\mathbf{K}_{n}$ manifest as separate spatial coordinates as illustrated in the bottom, right image of fig. 4 (b). This approach allows multiple LG modes to be generated and detected simultaneously producing a high data transmission rate.

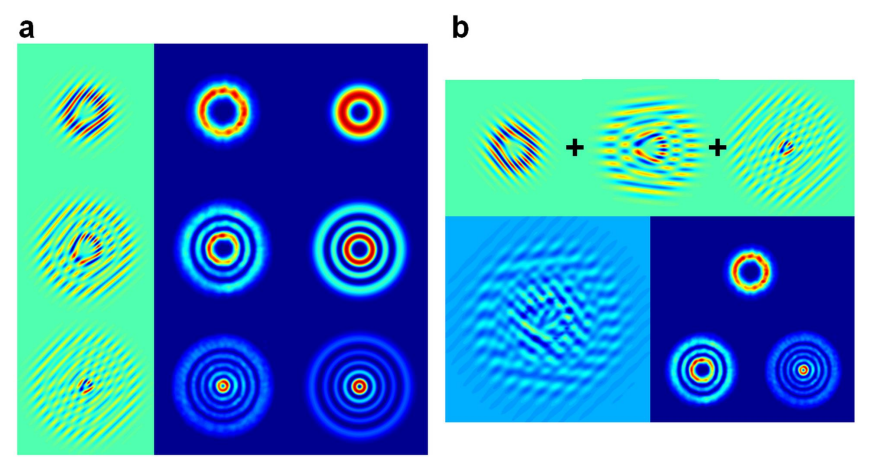

Figure 4. Complex-amplitude and spatial-multiplexed holograms. (a) Holograms encoded via complex-amplitude modulation for $\mathrm{LG}_{03}, \mathrm{LG}_{22}$ and $\mathrm{LG}_{41}$ with their corresponding experimental and theoretical intensity profiles. (b) Three LG holograms possessing different carrier frequencies are superimposed to obtain a multiplexed hologram (bottom, left) which, in the Fourier plane, produces the corresponding modes at three unique spatial positions (bottom, right). 


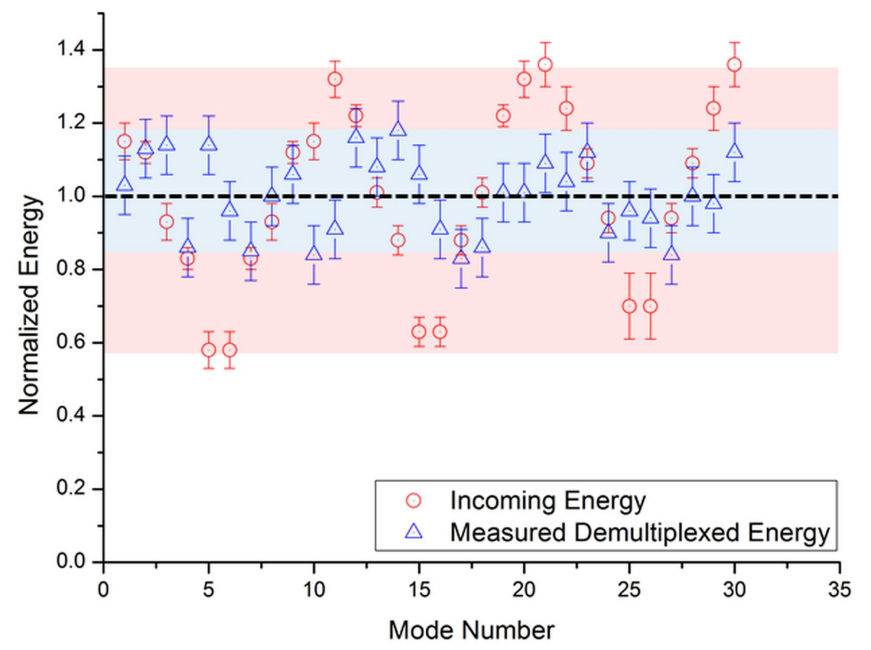

Figure 5. Normalization of detected signals. Plot of the the ratio of the energy in the generated modes with the energy in the detected signals as a function of the selected LG modes. The blue (red) data points contain the normalised (unnormalised) measured signals.

\section{SUPPLEMENTARY INFORMATION.}

\subsection{Normalization and Cross-Talk}

Since the encoding technique employed for creating these holograms requires that the amplitude of the field be normalized to unity, energy conservation is violated in the generated modes. To compensate for this power scaling, a correction parameter is introduced for each transmission function. The correction parameter, $\alpha_{n}$, is calculated as the ratio between the encoded optical field $\tilde{\Psi}_{n}(\mathbf{r})$ and the mode field $\Psi_{n}(\mathbf{r})$, where $\alpha_{n}=\max \left\{\left|\Psi_{n}(\mathbf{r})\right|\right\}^{-1}$ and $\alpha_{n} \in \Re^{+}$. In the case of the measurement procedure (i.e. performing the modal decomposition) the inner product can now be expressed as

$$
\left\langle\tilde{\Psi}_{n} \mid \tilde{\Psi}_{m}\right\rangle=\left\langle\alpha_{n} \Psi_{n} \mid \alpha_{m} \Psi_{m}\right\rangle=\alpha_{n} \alpha_{m} \delta_{n m}
$$

where the correction coefficients are determined as a special case of equation (3)

$$
\alpha_{n}^{2}=\left\langle\tilde{\Psi}_{n} \mid \tilde{\Psi}_{n}\right\rangle
$$

From the detection signal (i.e. the measured on-axis intensity, $I_{n}(\mathbf{r})$ ), the correction coefficients may be applied through the following relationship to normalise the signal to unit power:

$$
I_{n}(\mathbf{r})=\frac{\tilde{I}_{n}(\mathbf{r})}{\alpha_{n}^{2}}
$$

The normalization is illustrated in fig. 5 where the ratio between the energy of the generated mode and the energy of the demultiplexed signal is plotted for the various LG modes being used. The red data points contain the unnormalised measured signals illustrating a wider spread from unity (marked by the dotted line), while the blue data points contain the normalised signals which have a narrower spread.

The effect of the aperture size in the detection plane when performing a modal decomposition on the incoming modes was also investigated. The selected LG modes (used as information carriers) were generated on SLM-1 and decomposed via an inner-product measurement at SLM-2 (depicted in fig. 1). The measurement results are 
presented in fig. 6 which illustrate the (a) expected and measured cross-talk between neighbouring modes for a detection aperture size of (b) $24 \mu \mathrm{m}$ (c) $9.6 \mu \mathrm{m}$ and (d) $4.8 \mu \mathrm{m}$. In all cases [(b) - (d)] the strong diagonal and weak off-diagonal terms imply a highly accurate and precise measurement system which is unaffected by the size of the detection aperture.

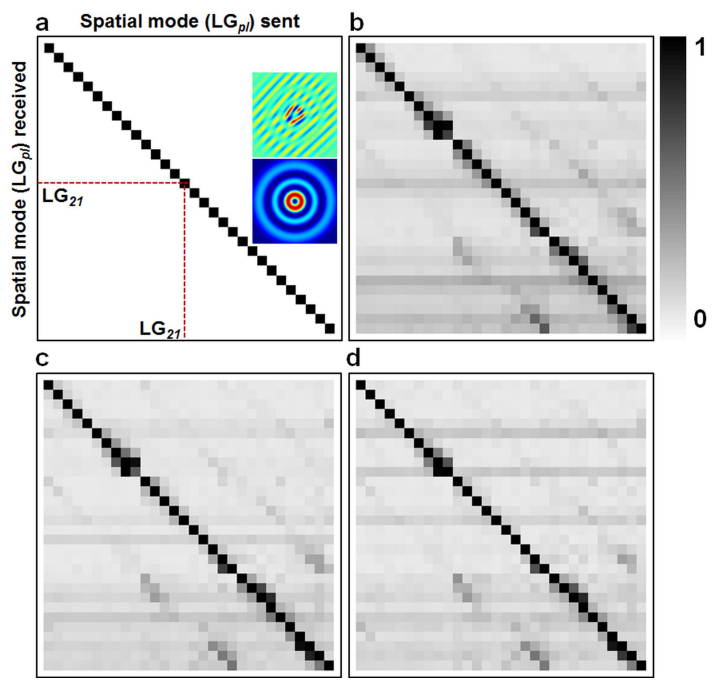

Figure 6. Effect of aperture size in the detection plane. Plot of the relative fractions of the intensity at each demultiplexed detector position for the selected LG modes. (a) The theoretical prediction and (b) - (d) measured crosstalk for aperture sizes of $24 \mu \mathrm{m}, 9.6 \mu \mathrm{m}$ and $4.8 \mu \mathrm{m}$, respectively.

\subsection{Gray-Scale and Colour}

The concept of assigning colour values present in a 2D image [as depicted in fig. 3 (b) and (c)] was initially tested with a simple image such as those in figs 7 (a) and 7 (c). This test required ensuring that the detector positions were aligned correctly with the on-axis demultiplexed signals. Incorrect alignment would result in incorrect colour values being decoded. The initial test images contain either 30 different gray-levels [7 (a)] or RGB-values $[7$ (c)]. In both cases it is evident that the reconstructed images are in very good agreement with the sent images illustrating the correct alignment of the demultiplex signals with the static detector positions. The bottom image of fig. 7 (c) contains a reconstructed image acquired four days after the system was initially aligned, illustrating its robustness and versatility. Additional gray-scale images [7 (b)] and RGB images [7 (c)], containing detailed features and higher resolution, were transmitted via this protocol demonstrating very high fidelity between the sent and received images.
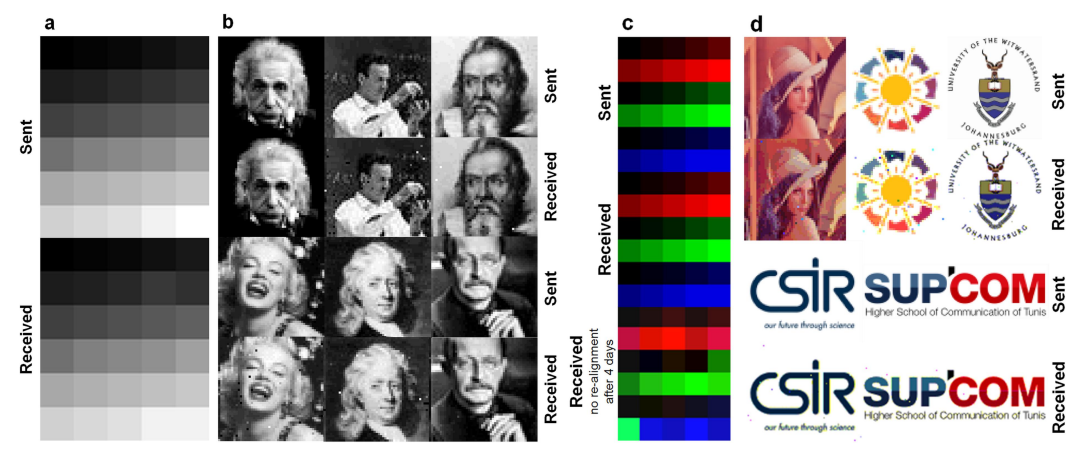

Figure 7. Gray-scale and RGB test. (a) The sent and received test image for verifying the success of the gray-scale encoding protocol. (b) Additional sent and received gray-scale images implemented with the gray-scale encoding scheme. (c) The sent and received test image for verifying the success of the RGB encoding protocol. (d) Additional sent and received colour images implemented with the RGB encoding scheme. 


\subsection{8-Bit Encoding}

In the gray-scale and RGB schemes the user is only concerned with detecting 1 signal out of a possible 35 . However, the 8-bit scheme involves detecting 256 unique combinations of either no signal incremented in unit steps up to all possible 8 signals. Since the on-axis intensity is higher for a single signal as opposed to all 8 signals being present [demonstrated in fig. 8 (a)], the user needs to carefully select the range of thresholds for the measured intensities. We investigated the impact the intensity threshold has on our encoding scheme and our findings are presented in fig. 8 (b). The red border marks the sent image and the green border the successfully reconstructed image obtained at a suitable threshold. The images in between denote the reconstructed images when the threshold was initially set too low illustrating either extreme or mild cross-talk with neighbouring gray-values. Most of the cross-talk occurs with the white colour-value because when the threshold is set too low, the detectors detect noise - often resulting in 8 signals being detected which is the trademark of the white colour-value [fig. 8 (a)].
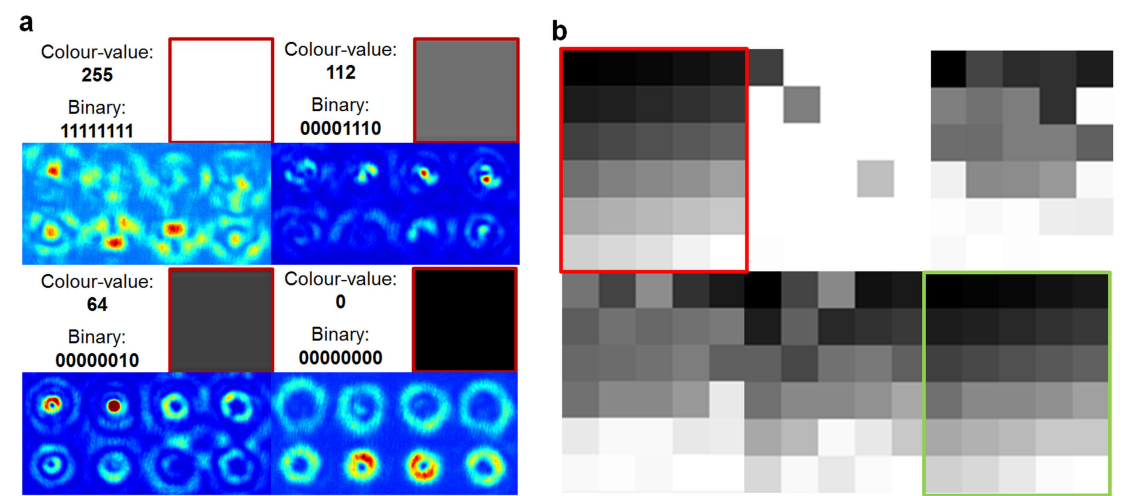

Figure 8. Threshold test for 8-bit encoding scheme. (a) Four selected gray-scales with their corresponding binary values and CCD images depicting the signals recorded at the detection plane. (b) The sent (red) and reconstructed gray-scale images for varying threshold values. Green marks successful reconstruction.

\section{Acknowledgments}

The authors would like to acknowledge support from the Claude Leon Foundation, the African Laser Centre (ALC), the ICTP affiliated centre The Optical Society of Tunisia, and CONACyT.

\section{REFERENCES}

[1] Gnauck, A. H., Winzer, P. J., Chandrasekhar, S., Liu, X., Zhu, B., and Peckham, D. W., "Spectrally efficient long-haul WDM transmission using 224-Gb/s polarization-multiplexed 16-QAM," J. Lightwave Technol. 29, 373-377 (Feb 2011).

[2] Zhou, X., Yu, J., Huang, M.-F., Shao, Y., Wang, T., Nelson, L., Magill, P., Birk, M., Borel, P. I., Peckham, D. W., Lingle, R., and Zhu, B., "64-Tb/s, 8 b/s/hz, PDM-36QAM transmission over $320 \mathrm{~km}$ using both pre- and post-transmission digital signal processing," J. Lightwave Technol. 29, 571-577 (Feb 2011).

[3] Wang, J., Li, S., Luo, M., Liu, J., Zhu, L., Li, C., Xie, D., Yang, Q., Yu, S., Sun, J., Zhang, X., Shieh, W., and Willner, A., "N-dimentional multiplexing link with 1.036-Pbit/s transmission capacity and 112.6$\mathrm{bit} / \mathrm{s} / \mathrm{Hz}$ spectral efficiency using OFDM-8QAM signals over 368 WDM pol-muxed 26 OAM modes," in [Optical Communication (ECOC), 2014 European Conference on], 1-3 (Sept 2014).

[4] Richardson, D. J., "Filling the light pipe," Science 330(6002), 327-328 (2010).

[5] Li, G., Bai, N., Zhao, N., and Xia, C., "Space-division multiplexing: the next frontier in optical communication," Adv. Opt. Photon. 6, 413-487 (Dec 2014). 
[6] Richardson, D. J., Fini, J. M., and Nelson, L. E., "Space-division multiplexing in optical fibres," Nat Photon 7, 354-362 (May 2013). Review.

[7] Ren, Y., Wang, Z., Xie, G., Li, L., Cao, Y., Liu, C., Liao, P., Yan, Y., Ahmed, N., Zhao, Z., Willner, A., Ashrafi, N., Ashrafi, S., Linquist, R. D., Bock, R., Tur, M., Molisch, A. F., and Willner, A. E., "Free-space optical communications using orbital-angular-momentum multiplexing combined with mimo-based spatial multiplexing," Opt. Lett. 40, 4210 (Sep 2015).

[8] Berdagué, S. and Facq, P., "Mode division multiplexing in optical fibers," Appl. Opt. 21(11), 1950 (1982).

[9] Li, G. and Liu, X., "Focus issue: Space multiplexed optical transmission," Opt. Express 19, 16574-16575 (Aug 2011).

[10] Luo, L.-W., Ophir, N., Chen, C. P., Gabrielli, L. H., Poitras, C. B., Bergmen, K., and Lipson, M., "WDMcompatible mode-division multiplexing on a silicon chip," Nat. Commun. 5, 3069 (2014).

[11] Milione, G., Lavery, M. P., Huang, H., Ren, Y., Xie, G., Nguyen, T. A., Karimi, E., Marrucci, L., Nolan, D. A., Alfano, R. R., et al., " $4 \times 20$ gbit/s mode division multiplexing over free space using vector modes and a q-plate mode (de) multiplexer," Opt. Lett. 40(9), 1980 (2015).

[12] Milione, G., Nguyen, T. A., Leach, J., Nolan, D. A., and Alfano, R. R., "Using the nonseparability of vector beams to encode information for optical communication," Opt. Lett. 40(21), 4887 (2015).

[13] Gibson, G., Courtial, J., Padgett, M. J., Vasnetsov, M., Pas'ko, V., Barnett, S. M., and Franke-Arnold, S., "Free-space information transfer using light beams carrying orbital angular momentum," Opt. Express 12, 5448 (Nov 2004).

[14] Bozinovic, N., Yue, Y., Ren, Y., Tur, M., Kristensen, P., Huang, H., Willner, A. E., and Ramachandran, S., "Terabit-scale orbital angular momentum mode division multiplexing in fibers," Science 340(6140), 1545-1548 (2013).

[15] Willner, A. E., Huang, H., Yan, Y., Ren, Y., Ahmed, N., Xie, G., Bao, C., Li, L., Cao, Y., Zhao, Z., Wang, J., Lavery, M. P. J., Tur, M., Ramachandran, S., Molisch, A. F., Ashrafi, N., and Ashrafi, S., "Optical communications using orbital angular momentum beams," Adv. Opt. Photon. 7(1), 66 (2015).

[16] Wang, J., Yang, J.-Y., Fazal, I. M., Ahmed, N., Yan, Y., Huang, H., Ren, Y., Yue, Y., Dolinar, S., Tur, M., and Willner, A. E., "Terabit free-space data transmission employing orbital angular momentum multiplexing," Nature Photon. 6(7), 488 (2012).

[17] Huang, H., Milione, G., Lavery, M. P. J., Xie, G., Ren, Y., Cao, Y., Ahmed, N., An Nguyen, T., Nolan, D. A., Li, M.-J., Tur, M., Alfano, R. R., and Willner, A. E., "Mode division multiplexing using an orbital angular momentum mode sorter and MIMO-DSP over a graded-index few-mode optical fibre.," Scientific reports 5(October), 14931 (2015).

[18] Brüning, R., Ndagano, B., McLaren, M., Schröter, S., Kobelke, J., Duparré, M., and Forbes, A., "Data transmission with twisted light through a free-space to fiber optical communication link," Journal of $O p$ tics 18(3), 03LT01 (2016).

[19] Yan, Y., Xie, G., Lavery, M. P. J., Huang, H., Ahmed, N., Bao, C., Ren, Y., Cao, Y., Li, L., Zhao, Z., Molisch, A. F., Tur, M., Padgett, M. J., and Willner, A. E., "High-capacity millimetre-wave communications with orbital angular momentum multiplexing.," Nat. Commun. 5, 4876 (2014).

[20] Torres, J. P., "Optical communications: Multiplexing twisted light," Nature Photonics 6(7), 420-422 (2012).

[21] Zhao, N., Li, X., Li, G., and Kahn, J. M., "Capacity limits of spatially multiplexed free-space communication," Nature Photon. 9(12), 822 (2015).

[22] Andersson, M., Berglind, E., and Björk, G., "Orbital angular momentum modes do not increase the channel capacity in communication links," New Journal of Physics 17(4), 43040 (2015).

[23] Forbes, A., Dudley, A., and McLaren, M., "Creation and detection of optical modes with spatial light modulators," Advances in Optics and Photonics 8(2), 200-227 (2016).

[24] Lei, T., Zhang, M., Li, Y., Jia, P., Liu, G. N., Xu, X., Li, Z., Min, C., Lin, J., Yu, C., Niu, H., and Yuan, X., "Massive individual orbital angular momentum channels for multiplexing enabled by Dammann gratings," Light: Science 83 Applications 4(3), e257 (2015).

[25] Li, S. and Wang, J., "Simultaneous demultiplexing and steering of multiple orbital angular momentum modes," Scientific Reports 5, 15406 EP - (Oct 2015). Article. 
[26] Rosales-Guzmán, C., Volke-Sepulveda, K., and Torres, J. P., "Light with enhanced optical chirality," Opt. Lett. 37, 3486-3488 (2012).

[27] Andrews, D. L., [Structured light and its applications], Academic Press (2008).

[28] Kuga, T., Torii, Y., Shiokawa, N., Hirano, T., Shimizu, Y., and Sasada, H., "Novel Optical Trap of Atoms with a Doughnut Beam," Phys. Rev. Lett 78(25), 4713-4716 (1997).

[29] Rosales-Guzmán, C., Hermosa, N., Belmonte, A., and Torres, J. P., "Experimental detection of transverse particle movement with structured light," Sci. Rep. 36(2815), 2815 (2013).

[30] Rosales-Guzmán, C., Hermosa, N., Belmonte, A., and Torres, J. P., "Direction-sensitive transverse velocity measurement by phase-modulated structured light beams," Opt. Lett. 18, 5415-5418 (2014).

[31] Rosales-Guzmán, C., Hermosa, N., Belmonte, A., and Torres, J. P., "Measuring the translational and rotational velocities of particles in helical motion using structured light," Opt. Express 22, 16504-16509 (2014).

[32] Belmonte, A., Rosales-Guzmán, C., and Torres, J. P., "Measurement of flow vorticity with helical beams of light," Optica 2, 1002-1005 (2015).

[33] Trichili, A., Rosales-guzmán, C., Dudley, A., Ndagano, B., Salem, A. B., Zghal, M., and Forbes, A., "Optical communication beyond orbital angular momentum," Sci. Rep. , 2-7 (2016).

[34] Litvin, I. A., Dudley, A., Roux, F. S., and Forbes, A., "Azimuthal decomposition with digital holograms," Opt. Express 20, 10996-11004 (May 2012).

[35] Dudley, A., Mhlanga, T., Lavery, M., McDonald, A., Roux, F. S., Padgett, M., and Forbes, A., "Efficient sorting of Bessel beams," Opt. Express 21, 165-171 (Jan 2013).

[36] Arrizón, V., Ruiz, U., Carrada, R., and González, L. A., "Pixelated phase computer holograms for the accurate encoding of scalar complex fields," J. Opt. Soc. Am. A 24, 3500-3507 (Nov 2007). 